\title{
Co-liquefaction of rubber seed and low rank coal: effect of weight ratio and temperature
}

\author{
S. N. A. M. Hassan ${ }^{1,2}$, M. A. M. Ishak ${ }^{2} \&$ K. Ismail ${ }^{1}$ \\ ${ }^{1}$ Faculty of Applied Sciences, Universiti Teknologi MARA, Malaysia \\ ${ }^{2}$ Faculty of Applied Sciences, Universiti Teknologi MARA Perlis, \\ Malaysia
}

\begin{abstract}
Increasing energy demands and limited petroleum reserves has seriously threatened economic development and social safety. The co-liquefaction of coal and biomass is one of the possible methods to solve the shortage of petroleum reserve. In this study, the co-liquefaction of Mukah Balingian (MB) low rank coal and rubber seed (RS) was performed by using a $15 \mathrm{~mL}$ tubing reactor, with $5 \mathrm{~mL}$ tetralin as a solvent for 30 minutes in various feedstock ratios and temperatures. The study focused on the effect of the weight ratio of feedstock and temperature on conversion and products distribution. The results showed that the weight ratio and temperature have a significant influence on the conversion, oil production, asphaltene and preasphaltene obtained. The addition of RS promotes liquefaction of MB by formation of the most valuable constituents of oil. The conversion was at its peak at the weight ratio of 30:70 (MB:RS) with $\sim 70 \%$ and $~ 47 \%$ conversion and oil production, respectively. Under this condition, the conversion and oil production of co-liquefaction were relatively higher than the liquefaction of $\mathrm{MB}$ alone. The development of this technology is of great significance in both the efficient utilization of resources and the improvement of ecological environment. Keywords: co-liquefaction, rubber seed, coal, ratio.
\end{abstract}

\section{Introduction}

Owing to the fact that energy composition properties are rich in coal, poor in oil and little in gas, coal liquefaction to produce alternative petroleum is of great significance to energy security concern. Low-to-medium rank coals have the traits of low price, relatively large porosity and high reactivity, which benefit their 
utilization for direct liquefaction. A primary concern with coal utilization is the release of carbon dioxide, the second greatest contributor to the greenhouse effect. Studies interest in direct coal liquefaction (DCL) has intensified [1]. DCL is a technology to convert coal directly into transportation fuels and chemicals, which is conducted at high temperatures and hydrogen pressure in the presence of solvents. Because of the severe reaction conditions with higher hydrogen consumption, the operation cost of DCL increases greatly. This will eventually makes the cost of oil from DCL to be difficult to compete with that from crude oil and thus limiting the development of large scale application of DCL.

Renewable energy has become more important globally especially with the current fuel and economic crisis. Biomass such as rubber seed is a contemptible, abundant, the only renewable organic energy source, abundantly composed of hydrogen and environmentally friendly [2]. Direct liquefaction of biomass to convert into alternative transportation fuels has been paid more and more attentions. There are about 4.2 million tons of agricultural waste (translating into 2.1 million tons of standard coal), which can be used as biomass energy in Peninsular Malaysia every year. However, most of them are often being directly burned-up on the spot just for vacating the crop land and the biomass energy has not been used effectively. Because of the low contents of $\mathrm{S}$ and $\mathrm{N}$ in biomass, the formation of $\mathrm{SO}_{\mathrm{x}}$ and $\mathrm{NO}_{\mathrm{x}}$ during firing of biomass is poor. Cutback of $\mathrm{CO}_{2}$, one of the greenhouse effect gasses, in air is growing one of hot issues in the world [3]. The $\mathrm{CO}_{2}$ formed during combustion of biomass is equal to the needed amount of $\mathrm{CO}_{2}$ when biomass grows up, resulting in almost no net $\mathrm{CO}_{2}$ emission after firing of biomass. This carbon neutrality stems from the various carbon fixation pathways plants use to grow, synthesizing carbohydrates from atmospheric $\mathrm{CO}_{2}$. Therefore, development and utilization of biomass energy can not only provide an overcome to energy problems, but also decrease environmental problems. However, the source of biomass for liquefaction was usually affected by season, years or producing sites. In addition, biomass typically has a relatively larger distributing area comparing with its total amount [4]. All these factors enhance the difficulty in feedstock supply and limit its large scale utilization.

The method to solve the above mentioned problems encountered in the individual liquefaction of coal and biomass is by co-liquefaction of them, which can maintain the stable supply of materials. Co-liquefaction of coal and biomass waste in preparation of oil has gained increasing research interest as the process can make full use of hydrogen in biomass, thus reducing the hydrogen consumption and moderating the conditions of DCL, realizing the mild operation of DCL. The addition of biomass to a coal conversion process reduces the carbon footprint of that process because, from an atmospheric perspective, biomass thermal conversion is inherently carbon neutral. Moreover, the biomass can promote the thermal disintegration of coal macromolecular, thus alleviate the severe reaction condition [5]. Pyrolysis of coal and biomass is the first step in the mechanism of co-liquefaction. Co-liquefaction of coal and biomass is a free radical process. Pyrolysis form unstable free radical fragments, which will be stabilized by obtaining active hydrogen to form hydrogenation products with lower molecular weight compared to that of coal or biomass. Biomass can produce 
smaller molecular fragments to combine with large fractions produced from coal decomposition and to terminate the cross-linking reactions between them, which will decrease the yield of the residue during liquefaction. By this way, the yield of low molecular products increases and oil quality is enhanced [6].

Till today, very limited studies of co-liquefaction on coal and biomass have been carried out. Influencing factor on the mechanism is not entirely clear. Thus, an attempt should be made for co-liquefaction of low rank Malaysian coal and biomass waste such as rubber seed.

\section{Experimental details}

\subsection{Feedstocks}

All feeds were acquired locally. The low rank coals used in this study were a sub-bituminous Mukah Balingian coal from Sarawak, which were received in a bulk form. The biomass feedstock used was rubber seeds, supplied by local rubber plantation in raw form. The coal and biomass were pulverized and all feeds were separately sieved. The particle size feeds used in this study were $\sim 212 \mu \mathrm{m}$. Feeds were dried at $105^{\circ} \mathrm{C}$ for approximately $24 \mathrm{~h}$ and stored in an air-tight container. Feed ratios used in this study were 0:100, 30:70, 50:50, 70:30, 100:0 (MB:RS).

Proximate and ultimate analyses of the coals and biomass were conducted by using Thermogravimetric analyzer (TGA) and CHNS analyzer. The results from these analyses were presented in Table 1. Solvents used were tetralin, tetrahydrofuran (THF), hexane and toluene. Tetralin and tetrahydrofuran were analytical grade reagents, purchased from Merck. Hexane and toluene were industrial grade reagents, purchased from local supplier. All solvents were used without further purification.

\subsection{Experimental setup and procedure}

The co-liquefaction experiments were carried out in a $15 \mathrm{~mL}$ tubing reactor shaken by using an air vibrator. $1.0 \mathrm{~g}$ of dried coal or rubber seeds or a mixture of coal and RS was loaded into the reactor together with $5 \mathrm{~mL}$ of tetralin and a $5 \mathrm{~mm}$ diameter steel ball. Before the liquefaction experiment, the reactor was sealed. The reactor was placed into a furnace and heated to the desired temperature $(360,380$, $400,420^{\circ} \mathrm{C}$ ) and maintained for 30 minutes. Then, the reactor was quenched to ambient temperature in an ice bath. The liquefaction mixture in the reactor was transferred completely to a clean flask by washing with THF. The THF soluble yield was further separated while the THF insoluble yield was accounted as liquefaction residue.

\subsection{Separation of liquefaction product}

The liquefaction product was separated by Soxhlet solvent extraction with hexane, toluene, and THF in turn. The hexane insoluble but toluene soluble fraction was defined as asphaltene (As), and the toluene insoluble but THF soluble fraction 
was defined as preasphaltene (PAs). The liquefaction conversion of feedstock and yields of liquefied product were defined according to equations (1), (2) and (3):

$$
\begin{aligned}
& \text { Conversion }(\%)=\left(\mathrm{W}_{\mathrm{f}} \frac{-\mathrm{W}_{\mathrm{r}}}{\mathrm{W}_{\mathrm{f}}} \times 100 \%\right. \\
& \mathrm{W}_{\text {as }}(\%)=\underline{\mathrm{W}}_{\underline{\mathrm{W}}_{\mathrm{f}}} \times 100 \% \\
& \mathrm{~W}_{\mathrm{pas}}(\%)=\underline{\mathrm{W}}_{\underline{\mathrm{W}}_{\mathrm{f}}} \times 100 \%
\end{aligned}
$$

where $\mathrm{W}_{\mathrm{f}}$ is the mass of the feedstock; $\mathrm{W}_{\mathrm{r}}$ is the mass of residue, $\mathrm{W}_{\mathrm{ts}}$ is the mass of toluene soluble fraction; and $\mathrm{W}_{\mathrm{ti}}$ is the mass of the tetrahydrofuran soluble fraction.

\section{Results and discussion}

\subsection{Proximate and ultimate analyses}

Several potential benefits of these feeds may be seen from the proximate and ultimate analyses. It can be seen that the H:C mass ratio for $\mathrm{MB}$ was 1.05, relatively low with comparison to that of $\mathrm{RS}$ with 1.51 . The higher $\mathrm{H}: \mathrm{C}$ mass ratio of RS suggested that biomass can act as hydrogen donor to the liquefaction process [7] and improve the yields and quality of the liquid products [8]. This will result in higher yields of oil and gas for RS liquefaction [9].

Table 1: Proximate and ultimate analyses of MB and RS.

\begin{tabular}{lcl}
\hline Sample & MB & RS \\
\hline Proximate analysis (wt\%, dry basis) & \\
Volatile matter & 77.5 & 96.8 \\
Ash & 10.1 & 2.9 \\
Fixed carbon & 12.4 & 6.1 \\
Ultimate analysis (wt\%, dry basis) & \\
Carbon & 50.0 & 64.5 \\
Hydrogen & 4.4 & 8.2 \\
Nitrogen & 1.3 & 3.6 \\
Oxygen (by difference) & 44.1 & 23.4 \\
Sulfur & 0.2 & 0.3 \\
\hline
\end{tabular}

\subsection{Individual liquefaction of MB and RS}

It is necessary to investigate the individual liquefaction of $\mathrm{MB}$ and $\mathrm{RS}$ alone. Table 2 shows the liquefaction properties of $\mathrm{MB}$ and $\mathrm{RS}$ at $380^{\circ} \mathrm{C}$, reaction time of $30 \mathrm{~min}$ and tetralin of $5 \mathrm{~mL}$. At the same liquefaction temperature, the liquefaction conversion and oil and gas yield of RS was higher in comparison to MB simultaneously. The results suggested that RS has higher thermal conversion activity with comparison to MB coal. 
Table 2: Liquefaction conversion and products distribution of MB and RS.

\begin{tabular}{lcccc}
\hline $\begin{array}{c}\text { Ratio } \\
(\mathrm{MB}: \mathrm{RS})\end{array}$ & Conversion (\%) & Asphaltene (\%) & Preasphaltene (\%) & $\begin{array}{c}\text { Oil + } \\
\text { Gas (\%) }\end{array}$ \\
\hline $0: 100$ & 86.6 & $33.3^{*}$ & $7.0^{* *}$ & 46.3 \\
$100: 0$ & 38.4 & 14.0 & 6.2 & 18.2 \\
\hline * toluene soluble & & & & \\
** tetrahydrofuran soluble & & &
\end{tabular}

\subsection{Effect of blending ratio}

Researchers suggested that the amount of biomass blending has a great influence on the co-liquefaction of coal and biomass. The co-liquefaction was conducted at $380^{\circ} \mathrm{C}$ and $30 \mathrm{~min}$, and the results are shown in Figure 1. The percent conversion increased from $38.4 \%$ with $100: 0$ blending ratio to $86.6 \%$ with a $0: 100$ blending ratio of MB:RS. Apparently, the light products of oil and gas increased greatly, with heavy products As and PAs hardly changed with the increasing ratio of RS. At 30:70 blending ratio, the conversion was $\sim 70.0 \%$, with As and Pas at $13.7 \%$ and $9.3 \%$ respectively. The conversion increases with the increasing amount of RS blending; 70:30 (48.6\%), 50:50 (59.2\%) and 30:70 (70\%). The highest oil +gas yield was at the $30: 70$ blending ratio with $46.9 \%$. This is likely due to the fact that $\mathrm{RS}$ is more easily to be liquefied and gives more light products in comparison to coal.

The increase in conversion mainly contributed to the increase in oil and gas. This means that in the co-liquefaction process of RS and MB blends, RS is beneficial for promoting the formation of oil, because it can act as hydrogendonors to prevent the retrogressive reactions [10].

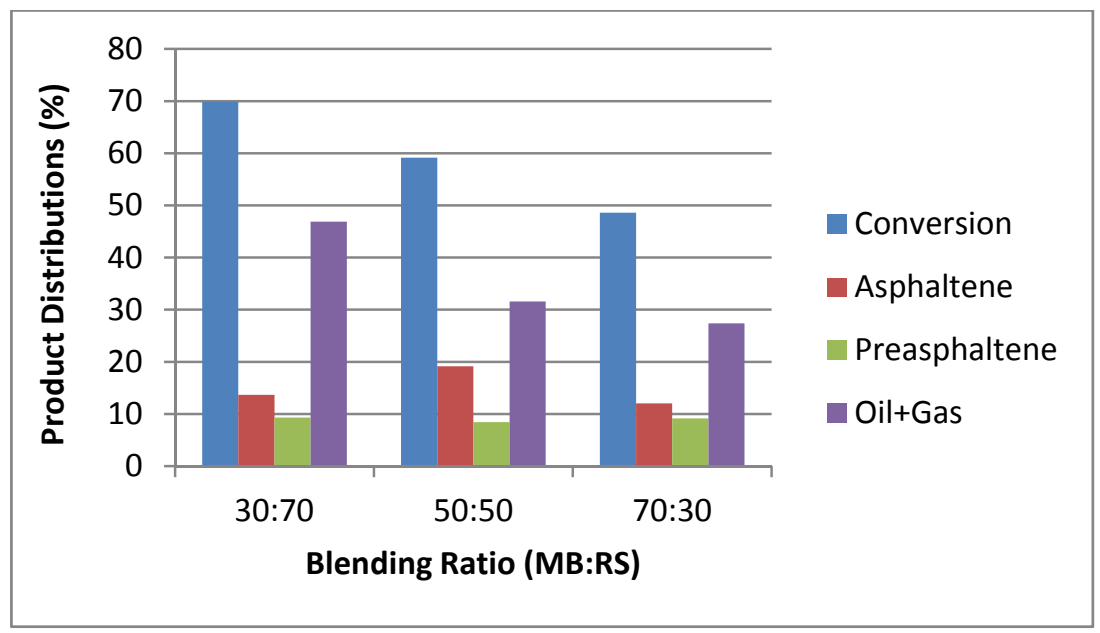

Figure 1: Effect of blending ratios on products distribution (conditions; temperature: $380^{\circ} \mathrm{C}$, reaction time: 30 minutes). 


\subsection{Effect of temperature}

Temperature affects the co-liquefaction behaviors of MB coal and RS. It has been shown that co-liquefaction of $\mathrm{MB}$ coal and RS at 30:70 blending ratio gave the largest synergistic effect that promotes high percent of conversion and oil+gas yields. The effect of temperature on the co-liquefaction properties of MB coal and $\mathrm{RS}$ at 30:70 blending ratio of MB:RS with $30 \mathrm{~min}$ was further investigated. Generally, $300-450^{\circ} \mathrm{C}$ is the temperature range where the decomposition of coal and rubber seed occurs, so several points from 360 to $420^{\circ} \mathrm{C}$ were selected in this work. The results were shown in Figure 2. From Figure 2 it shows that by increasing temperature from 360 to $420^{\circ} \mathrm{C}$, the percent conversion slightly increase from $66.7 \%$ to $71.9 \%$. The first step in co-liquefaction is pyrolysis and the main factor that affects the pyrolysis is greatly influenced by temperature. At low temperature, the main reaction is thermolysis of coal and biomass into As and PAs with large molecular weights, which will further be pyrolyzed into smaller free radicals or intermediates with increasing temperature [11]. The free radicals or intermediates can be stabilized by capturing active hydrogen to form oil + gas compositions.

As temperature increase, the oil + gas yield fluctuates from $33.8 \%$ to $42.0 \%$ with the lowest at $400^{\circ} \mathrm{C}$. The yield of As+PAs reached the maximum value at $400^{\circ} \mathrm{C}$ with $36.8 \%$ and then decreased. This behaviour is believed to have resulted from the following reaction:

$$
\text { Coal }+ \text { rubber seed }->\text { preasphaltene }->\text { asphaltene }->\text { oil }+ \text { gas }
$$

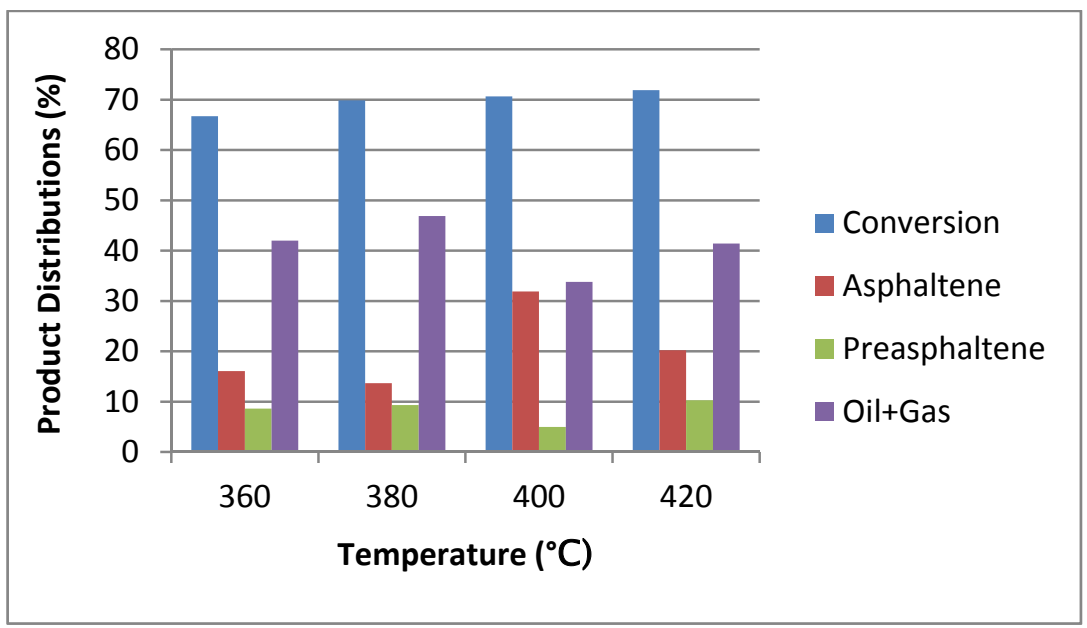

Figure 2: Effect of temperature on product distributions (conditions; blending ratio: $30: 70$, reaction time: 30 minutes).

When the temperature decreased below $400^{\circ} \mathrm{C}$, the formation rate of As+PAs was faster than that of its decomposition to oil+gas. This observation indicates that reaction at low temperature was dominated by cracking reaction from reactant to 
macromolecular As+PAs. The yield of As+PAs increases with the increase of temperature. As the temperature further increase, the decomposition of As+PAs to form free radicals hydrogenated to produce oil become the predominant reactions, leading to the reduction of As+PAs yield. The consumption of hydrogen increases with the increase of temperature, and this is mainly due to the faster cracking of coal molecules, and thus resulted in more active hydrogen consumption.

\section{Conclusions}

Co-liquefaction of rubber seed blends with sub-bituminous Mukah Balingian low rank coal at several feed ratios has shown significant results. Rubber seed has higher liquefaction activity than Mukah Balingian coal. Under the same liquefaction conditions, rubber seed gave higher conversion and oil and gas yield in comparison to Mukah Balingian coal. Co-liquefaction process was influenced by the blending ratio and temperature. The largest enhancements in conversion and oil and gas yield were obtained at 30:70 (MB:RS) blending ratio.

\section{References}

[1] Sirin, M., Somkiat, N., Pattarapan P., Improvement of oil yield and its distribution from coal extraction using sulfide catalysts. Fuel, 86, pp. 2485-2490, 2007.

[2] Yigang, C., Chao W., Weipeng, L., Zhengyu, Y., Study of the co-deoxyliquefaction of biomass and vegetable oil for hydrocarbon oil production. Bioresource Technology, 101, pp. 4600-4607, 2010.

[3] Hengfu, S., Qingqing, J., Zhengyi, C., Zhicai, W., Zhiping, L., Shibiao, R., Chunxiu, P., Co-liquefaction of rice straw and coal using different catalysts. Fuel, 109, pp. 9-13, 2013.

[4] Hengfu, S., Chuanjun, S., Zhengyi, C., Zhicai, W., Zhiping, L., Shibiao, R., Chunxiu, P., Haiping, L., Co-liquefaction behavior of a sub-bituminous coal and sawdust. Energy, 36, pp. 6645-6650, 2011.

[5] Mohd, F.A., Zaidi, A.G., Mohd, A.M.I., Khudzir, I., Co-liquefaction of Low Rank Malaysian Coal and Palm Kernel Shell - The Effect of Temperature. IEEE Colloquium on Humanities, Science \& Engineering Research (CHUSER), pp. 627-630, 2012.

[6] Zhenxing, G., Zongqing, B., Jin, B., Zhiqing, W., Wen, L., Co-liquefaction of lignite and sawdust under syngas. Fuel Processing Technology, 92, pp. 119-125, 2011.

[7] Ryan, M.S., Nicholas, C.M., Nathan, T.W., Co-pyrolysis of low rank coals and biomass: Product distributions. Fuels, 112, pp. 74-82, 2013.

[8] Hengfu, S., Zhengyi, C., Chunbao, C.X., Recent advance in direct coal liquefaction. Energies, 3, pp. 155-170, 2010.

[9] Hassan, S.N.A.M., Ishak, M.A.M., Ismail, K., Ali, S.N., Yusop, M.F., Comparison Study of Rubber Seed Shell and Kernel (Hevea brasiliensis) as Raw Material for Bio-Oil Production. Energy Procedia, 52, pp. 610-617, 2013. 
518 Energy and Sustainability V

[10] Zhou, H., Cai, Z., Shui, H., Lei, Z., Wang, Z., Li, H., Co-liquefaction properties of Shenfu coal and rice straw. Journal of Fuel Chemistry and Technology, 39(10), pp. 721-727, 2011.

[11] Qingjie, T., Zhihong, W., The effect of technological parameter on the co-liquefaction of coal with lignin. Advance Materials Research, 577, pp. 167-170, 2012. 